\title{
Le Registre suisse du cancer de l'enfant: expériences comme Registre national du cancer
}

\author{
Claudia E. Kuehnia, \\ Gisela Michela, \\ Matthias Egger a, \\ Marcel Zwahlen ${ }^{a}$ \\ Maja Beck Popovic, \\ Felix Niggli ${ }^{b}$ \\ Nicolas X. von der Weidc
}

Pour le Groupe d'oncologie pédiatrique suisse $(S P O G)^{*}$ et le Registre suisse du cancer de l'enfant (RSCE)*

a Registre suisse du cancer de l'enfant, Institut de médecine sociale et préventive, Université de Berne

b Président, Groupe d'oncologie pédiatrique suisse, Universitätkinderklinik, Zurich

c Ancien président, Groupe d'oncologie pédiatrique suisse, Universitätskinderspital UKBB, Bâle

d Médecin chef, unité d'hémato-oncologie pédiatrique, CHUV, Lausanne

\footnotetext{
* Les membres des groupes et les références se trouveront sous www.bullmed.ch $\rightarrow$ Numéro actuel ou Archives $\rightarrow 2013 \rightarrow 9$.
}

Correspondance:

Prof. Dr Claudia E. Kuehni Institut de médecine sociale et préventive

Finkenhubelweg 11

$\mathrm{CH}-3012$ Berne

Tél. 0316313507

Fax 0316313520

kuehni[at]ispm.unibe.ch

\section{Introduction}

Dans le cadre de la procédure de consultation de la nouvelle loi fédérale sur l'enregistrement du cancer, les avantages et les inconvénients d'un tel enregistrement au niveau national font à nouveau l'objet de discussions en Suisse. Le registre suisse du cancer de l'enfant (RSCE) possède une expérience de bientôt 40 ans en tant que registre national du cancer chez les enfants et les adolescents. Le présent article explique les particularités des cancers pédiatriques, décrit l'expérience du RSCE en tant que registre national du cancer et montre l'importance des données récoltées pour la clinique, la recherche et la santé publique.

\section{Comment fonctionne le Registre suisse du cancer de l'enfant?}

Le RSCE a été fondé en 1976 par le Groupe d'oncologie pédiatrique suisse (SPOG). Il est depuis lors la seule plateforme nationale d'enregistrement des maladies oncologiques en Suisse [1,2] ${ }^{*}$. Les maladies oncologiques adultes sont d'abord enregistrées au niveau cantonal. Une couverture nationale ne sera mise en place qu'à partir de 2013 au mieux [3,4]. Le RSCE est similaire aux registres nationaux des cancers de l'enfant dans d'autres pays, à savoir que c'est à la fois un registre épidémiologique populationnel pour le monitoring des cancers de l'enfant et un registre clinique collectant des données sur leur traitement et l'évolution de la maladie.

Sont collectées les données de toutes les personnes de 0 à 20 ans résidant en Suisse chez qui une leucémie, une tumeur cérébrale bénigne ou maligne, une tumeur solide maligne ou une histiocytose a été diagnostiquée [5]. Pour ces cas, des données supplémentaires sont collectées auprès des cliniques qui les prennent en charge, auprès des laboratoires de pathologie et auprès d'autres registres tel que ceux des hôpitaux ou de la statistique suisse de la mortalité Une mise à niveau de ces données est effectuée régulièrement avec les registres cantonaux du cancer. Les enfants entre 0 et 15 ans sont enregistrés de manière presque complète (>95\%). Les personnes développant un cancer entre 15 et 20 ans n'ont été que partiellement enregistrés jusqu'ici (env. 60-70\%). Les maladies sont codées au moyen de la classification internationale des maladies oncologiques ( $3^{\mathrm{e}}$ révision, CID-O-3) et de la classification internationale des cancers de l'enfant (3e révision, ICCC-3) [6].

Les données de base sur la maladie et son traitement par rapport sont fournies peu après le diagnos- tic, par les médecins traitants (SPOG), sur la base du dossier médical. L'évolution en cours de traitement est enregistrée de façon régulière, toujours sur la base dossier médical. Par la suite, la survie du patient est notifiée grâce à un recoupement avec les registres des habitants des communes et les statistiques de mortalité. Les tumeurs secondaires sont répertoriées grâce à un recoupement avec les registres cantonaux du cancer. L'étude suisse des survivants du cancer de l'enfant (Swiss Childhood Cancer Survivor Study) permet d'analyser les morbidités et la qualité de vie à long terme des patients guéris [7].

Protection des données: à l'instar des registres cantonaux, le RSCE possède une autorisation générale pour les Registres, donnée par la Commission fédérale d'experts du secret professionnel en matière de recherche médicale [2]. Cette autorisation permet la collecte généralisée de données non-anonymisées sur les cancers pédiatriques en Suisse provenant de différentes sources, ainsi qu'une synchronisation de ces données avec la statistique suisse de la mortalité et les registres cantonaux du cancer. Les médecins traitants sont tenus d'informer les patients et leurs parents sur l'existence du Registre. Un consentement éclairé écrit n'est pas obligatoire. Toutefois, les familles possèdent un droit de véto. Ce droit d'opposition n'est utilisé que très rarement dans notre expérience: plus de $98 \%$ des personnes contactées autorisent la collecte de leur données, rendant possible un enregistrement généralisé de données représentatives. Le RSCE reste sous la surveillance des préposés cantonaux et nationaux à la protection des données et doit observer des lignes directrices strictes concernant celle-ci. Les données personnelles (nom, adresse) et les données cliniques sont enregistrées sur des bases de données strictement séparées.

Financement: le Registre suisse du cancer de l'enfant se bat en permanence pour survivre financièrement, étant donné qu'il ne reçoit aucune aide régulière de l'Etat fédéral ou des cantons. Jusqu'à maintenant, il a été subventionné par le SPOG et l'Institut de médecine sociale et préventive de l'université de Berne ainsi que par les dons d'associations de parents et de personnes privées. Ces moyens ne suffiront malheureusement pas à conserver le haut niveau de qualité atteint et à remplir les exigences à venir en matière de protection des données. Jusqu'à l'entrée en vigueur de la loi sur l'enregistrement du cancer, la Conférence suisse des directrices et directeurs cantonaux de la santé (CDS) apporte une contribution annuelle bienvenue. 
Objectifs du Registre suisse du cancer de l'enfant

- Collection de données populationnelles représentatives sur les maladies oncologiques de l'enfant et l'adolescent en Suisse (incidence, prévalence, tendances temporelles, répartition géographique et taux de survie);

- Documentation du diagnostic précis, du traitement, de l'inclusion dans une étude clinique;

- Documentation du pronostic à court et long terme des cancers de l'enfant et de l'adolescent (rémissions, rechute, survie, complications à long terme, séquelles);

- Création d'une plateforme de recherche pour des études cliniques, épidémiologiques et de recherche fondamentale.

Il contribue à travers cela à...

- une amélioration constante du traitement;

- la planification de la prise en charge en oncologie pédiatrique;

- I'identification des séquelles dues au traitement, avec comme objectif de pouvoir les prendre en charge précocement et les prévenir dans le futur;

- l'exploration des étiologies possibles des maladies oncologiques de l'enfant et l'adolescent. relative des maladies oncologiques pédiatriques, une expertise particulière est requise pour en garantir un diagnostic correct, un traitement efficace et une bonne documentation de leur évolution [9]. Ceci n'est possible que si les oncologues pédiatres travaillent en étroite collaboration (à l'intérieur du SPOG) et que les données sont centralisées et traitées de manière uniforme (Registre suisse du cancer de l'enfant).

Les traitements du cancer de l'enfant diffèrent également de ceux des adultes. On peut donner aux enfants les agents cytostatiques appropriés à des dosages plus élevés. Par contre, il faut être particulièrement attentif aux organes encore en croissance (le système nerveux central ou les organes reproducteurs, par ex.). C'est pour cela, entre autre, que les leucémies lymphoblastiques aiguës ont des schémas thérapeutiques différents chez l'enfant et l'adulte, pour ne citer que cet exemple.

Actuellement, plus de $80 \%$ des enfants atteints peuvent être guéris [10] mais parmi ceux-ci, deux tiers vont développer des complications à long terme (problèmes endocriniens, psychiques, surdité, cécité, problèmes de fertilité, maladies cardiovasculaires et pulmonaires ou cancers secondaires) qui peuvent apparaître des années voire des dizaines d'années après la guérison [11,12]. C'est pourquoi il est important que l'observation clinique et la recherche ne s'arrêtent pas 5 ou 10 ans après la fin du traitement. Même des dizaines d'années plus tard, les complications doivent pouvoir être reconnues, afin d'une part de les traiter précocement et d'autre part d'essayer de prévenir leur apparition chez les patients futurs [13]. C'est pour cela que le Registre du cancer de l'enfant effectue un suivi sur toute la vie. Parallèlement, des programmes de suivi clinique des survivants devront être mis en place.

\section{Quels sont les résultats du RSCE?}

1. Les maladies oncologiques pédiatriques sont répertoriées de manière généralisée et systématique en Suisse. Plus de 8700 malades ont été enregistrés jusqu'à présent. L'incidence du cancer de l'enfant en Suisse est de 15,7 nouveaux cas pour 100000 personnes/an et est similaire à celle des pays voisins [5]. Le monitoring effectué depuis 40 ans permet d'évaluer la variation de l'incidence au fil des ans, pour voir par exemple si des conditions environnementales ou le mode de vie pouvaient avoir une influence. La figure 1 montre qu'au cours des 20 dernières années, cette incidence est restée stable [5]. L'incidence du cancer chez l'adulte en Suisse n'a été jusqu'à présent qu'extrapolée sur la base des chiffres des registres cantonaux existants [3]. La nouvelle loi devrait apporter une solution nationale, aussi pour les adultes.

2. La collaboration étroite avec le réseau suisse des cliniques d'oncologie pédiatrique (SPOG) permet d'avoir une vue d'ensemble sur la prise en charge, les taux de guérison et les complications à long terme. Tous les patients sont pris en charge selon des protocoles
Alors que chez les adultes les carcinomes prédominent, les enfants souffrent principalement de leucémies, de tumeurs cérébrales, de tumeurs em bryonnaires (rétino-, neuro-, néphro- et hépatoblastome ainsi que tumeurs germinales) et de sarcomes [3]. En raison de la grande diversité et de la rareté 
standardisés, régulièrement actualisés. La majorité d'entre eux sont inscrits sur des études cliniques internationales multicentriques, dites d'optimisation thérapeutique. Le Registre suisse du cancer de l'enfant est à même de documenter l'amélioration impressionnante des taux de guérison grâce à ce type de stratégie: les taux de survie à 10 ans pour les enfants atteints de cancer sont passés de $58 \%$ dans les années 70 à $82 \%$ depuis 2002 (fig. 2) [5].

3. Le suivi durant toute la vie permet un monitoring de la santé de l'enfant. La mortalité est enregistrée grâce à un recoupement avec le registre des habitants des communes. Dans le cadre de l'étude de cohorte nationale (Swiss Childhood Cancer Survivor Study) basée sur les données du Registre, un questionnaire est envoyé aux survivants et investigue leur qualité de vie, les complications somatiques et psychiques éventuelles, les habitudes de vie et le suivi médical ambulatoire [7]. Pour exemple, cette étude a montré que dans l'ensemble, les jeunes adultes survivants avaient une bonne qualité de vie et une bonne santé psychique [14-16]. Elle a permis également identifier des groupes plus à risque de développer des complications tardives $[15,17]$. En se basant sur ces nouvelles connaissances, le traitement et le suivi ambulatoire peuvent être améliorés pour les nouveaux patients ainsi que pour les anciens.

4. Les données détaillées rassemblées concernant la prise en charge médicale aident à la planification future dans le domaine de l'oncologie pédiatrique, en ambulatoire comme en hospitalisation. Par exemple la planification des lits de soins aigus, des centres de transplantation, du suivi ambulatoire et de la transition des soins pédiatriques vers les soins adultes. La Swiss Childhood Cancer Survivor Study a révélé des lacunes significatives dans le suivi

Figure 1

Taux d'incidence (nombre de nouvelles maladies/100000 enfants/an) des maladies oncologiques pédiatriques en Suisse entre 1992-2011. Age au diagnostic 0-14 ans, N = 3696 .

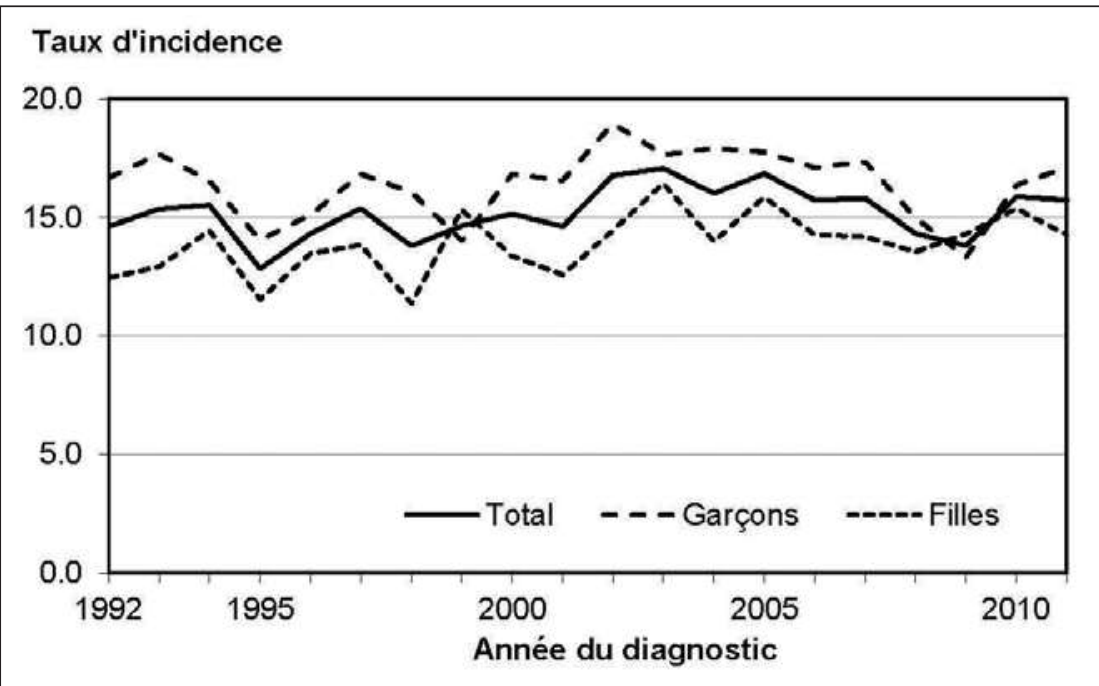

des patients, dès qu'ils sortaient de l'encadrement pédiatrique $[18,19]$. Des programmes de recherche en collaboration avec les oncologues pédiatres sont en cours afin de définir les causes et de trouver des solutions à ce problème.

5. Les questions actuelles sur le rôle potentiel de l'environnement sur la genèse des cancers peuvent être abordées rapidement grâce au Registre suisse du cancer de l'enfant. L'étude CANUPIS en est un bon exemple [20]. Elle a été demandée par le Conseil fédéral et le Parlement après qu'une étude allemande ait démontré un risque de leucémies 2 fois plus élevé chez les enfants en bas âge vivant à proximité d'une centrale nucléaire dans ce pays. Grâce à sa base de données complète à l'échelon national, le RSCE a pu, en collaboration avec la cohorte nationale suisse (www.swissnationalcohort.ch), répondre très rapidement à cette question politiquement brûlante (www.canupis.ch). Les résultats ont montré qu'il n'y avait pas de risque significativement plus élevé pour les enfants vivant près des centrales nucléaires en Suisse [20]. Le RSCE participe également à une étude internationale, CEFALO, qui a pour but d'évaluer l'influence de l'utilisation des téléphones portables sur le risque de tumeur cérébrale chez l'enfant. Ici aussi, les résultats se sont révélés rassurants [21].

\section{Comment les cancers de l'enfant sont-ils enregistrés dans les pays voisins?}

La situation est similaire à la Suisse en Allemagne, en France et en Angleterre. Dans ces pays, les données sur les cancers de l'enfant sont répertoriées par des registres spécialisés qui collectent également des détails sur les thérapies et le suivi à long terme. Le registre allemand du cancer de l'enfant (www. kinderkrebsregister.de) [22], le «Registre National des Tumeurs Solides de l'Enfant» et le «Registre national des hémopathies malignes de l'Enfant» en France [23], de même que le «Registre national des tumeurs de l'enfant» à Oxford [24] ont tous développé un programme de recherche spécialisé qui aborde la question de l'étiologie, du traitement et du pronostic à long terme des cancers de l'enfant. Le RSCE travaille en étroite collaboration avec ces registres. Dans ces 3 pays, des registres régionaux du cancer existent en parallèle au registre national. Ils collectent les incidences et les données sur la survie pour toutes les catégories d'âge.

\section{La nouvelle loi fédérale prévue pour l'enre- gistrement du cancer et ses conséquences pour l'enregistrement des cancers de l'enfant} En 2010, le conseil fédéral a chargé le Département fédéral de l'intérieur de travailler sur un avant-projet de loi fédérale concernant l'enregistrement du cancer. La nouvelle loi fédérale doit poser les bases d'une harmonisation des conditions générales d'enregistrement du cancer en Suisse, actuellement juridiquement très diverses selon les cantons. En outre, 
Figure 2

Modification du taux de survie des enfants atteints de cancer en Suisse depuis 1976, (année du diagnostic [Dx] 1976-2011).

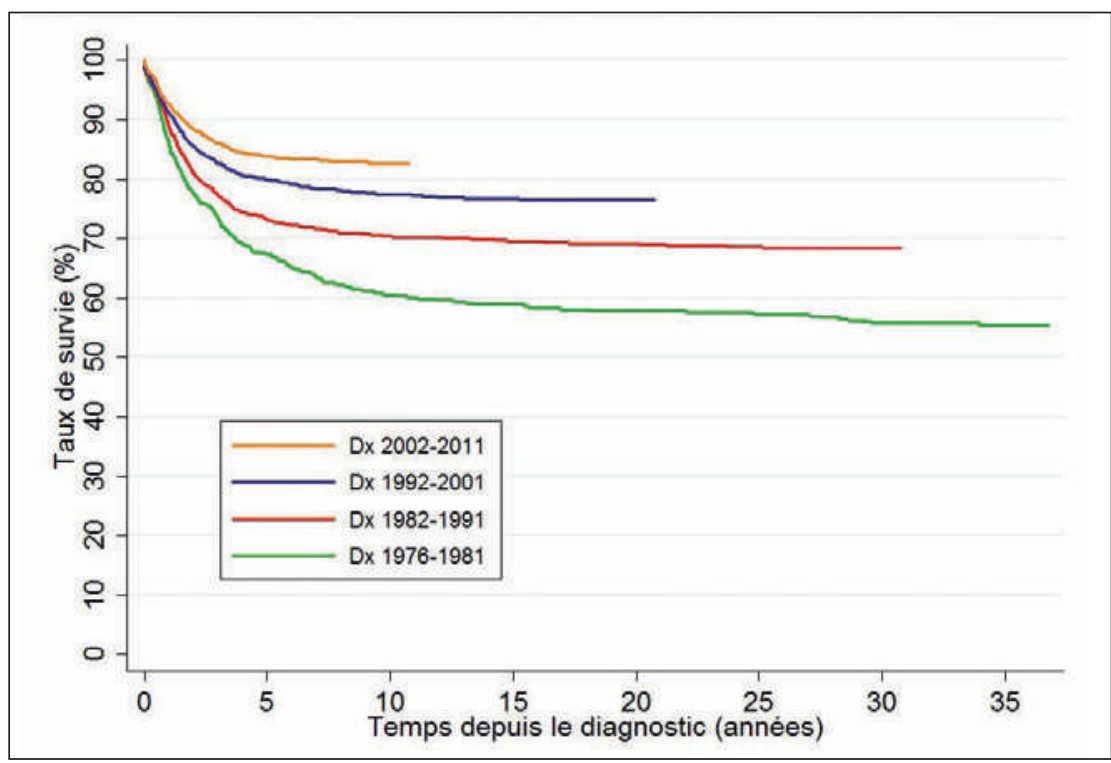

elle doit permettre la saisie au niveau suisse de tous les nouveaux cas de cancer et de rassembler les données pertinentes sur leur évolution. L'avant-projet de loi est en consultation jusqu'au 22 mars 2013 (www.bag.admin.ch/themen/gesundheitspolitik/ 10374/index.html?lang=fr).

La nouvelle réglementation sera basée sur les structures existantes d'enregistrement du cancer. Cet enregistrement est effectué actuellement, pour les tumeurs des adultes, par les registres cantonaux et régionaux du cancer. Les maladies oncologiques de l'enfant et de l'adolescent continueront à être enregistrées par le Registre suisse du cancer de l'enfant. De plus, la Confédération soutiendra un Centre national pour l'enregistrement et l'épidémiologie du cancer, responsable de la mise en commun des données cantonales et régionales, du traitement et de l'analyse centralisés des données et disposant d'un service ad hoc de cryptage des données.

La nouvelle loi devrait apporter des améliorations importantes: 1) La protection des données et l'obligation pour les médecins de les transmettre aux Registres sera réglementée au niveau national. La validation des données personnelles essentielles comme le lieu de domicile, l'adresse et le statut vital qui garantissent les fonctions centrales d'un registre du cancer (incidence et survie) sera simplifiée, moins coûteuse et se fera avec une meilleure protection de ces données. 2) L'apparition d'une seconde ou troisième tumeur chez le même patient pourra être identifiée quelque soit l'âge du patient au moment du diagnostic de la première tumeur. Actuellement ceci n'est le cas de manière fiable en Suisse que pour les enfants diagnostiqués avant 20 ans: les adultes ne sont plus identifiables à partir du moment où ils changent de canton. 3) Le financement de l'enregistrement du cancer chez l'adulte et l'enfant sera enfin réglé de façon satisfaisante. Quelques points du présent avantprojet peuvent néanmoins être améliorés, par ex. l'anonymisation prévue de toutes les données 10 ans après la mort du patient. Celle-ci pourrait entraver fortement la recherche sur les causes et les traitements du cancer. Notamment en ce qui concerne les cancers rares, pour lesquels il est important de pouvoir garder l'accès aux données de tous les cas, même ceux qui datent de plus de 10 ans. Après anonymisation, les données ne peuvent être utilisées pour la recherche que sous certaines conditions. De plus, l'élimination sélective des données des patients décédés conduirait à une falsification systématique (biais) des résultats.

La projet de loi prévoit que seules des données de base très limitées (minimal data set) puissent être rassemblées sans consentement mais avec toutefois un droit de véto du patient. Les données sortant de ce cadre, par ex. déjà celles concernant le traitement, nécessiteront dans le futur un consentement éclairé écrit. Ceci représenterait un grand pas en arrière par rapport à la réglementation actuelle. En règle générale, ce que les patients ne souhaitent pas, c'est justement l'enregistrement du diagnostic de leur cancer (donc le minimal data set). Il n'est encore jamais arrivé jusqu'à maintenant, dans le Registre suisse du cancer de l'enfant, que des patients ou leurs parents qui étaient d'accord pour la collecte du minimal data set émettent une objection à la collecte des données complémentaires concernant leur traitement ou le cours de leur maladie. L'obtention d'un consentement éclairé peut être considérée comme une activité de relative routine pour les grands hôpitaux. Dans les plus petites structures qui ne s'occupent qu'occasionnellement de patients atteints de cancer, l'introduction d'une telle obligation serait difficile à mettre en place de manière efficiente. On y renoncerait ainsi probablement dans la majorité des cas, ce qui conduirait à une situation anarchique, où un contrôle de la qualité du traitement ne pourrait être effectuée que pour les patients traités dans les grands hôpitaux. Une analyse complète et systématique du devenir des tous les patients atteints de cancer dans notre pays deviendrait alors impossible.

\section{Conclusion}

Le Registre national suisse du cancer de l'enfant, avec ses presque 40 ans d'expérience, a fait ses preuves. Le recensement national centralisé et les liens étroits avec les cliniciens du SPOG permettent un enregistrement efficient et de haute qualité des cancers pédiatriques en Suisse; cette qualité serait perdue si l'enregistrement était fractionné au niveau cantonal ou régional. L'activité du Registre couplée à la collaboration étroite avec les médecins spécialistes a permis d'énormes progrès dans la maîtrise de ces maladies graves et a considérablement amélioré la situation des enfants et de leurs familles. C'est la raison pour laquelle nous nous réjouissons et saluons la nouvelle loi qui base sur ces acquis et en favorise les développements futurs. 


\section{Comité du SPOG}

Prof. Dr méd. Felix Niggli, président; Prof. Dr méd. Nicolas von der Weid, ancien président; Dr méd. Heinz Hengartner, vice-président; PD Dr méd. M. Beck-Popovic, membre.

Responsables des Centres du SPOG / Représentant(e)s du Conseil de Recherche du SPOG

Prof. Dr méd. R. Ammann, Berne; Dr méd. R. Angst, Aarau; PD Dr méd. M. Beck-Popovic, Lausanne; Dr méd. E. Bergstraesser, Zurich; Dr méd. P. Brazzola, Bellinzone; Dr méd. J. Greiner, St-Gall; Prof. Dr méd. M. Grotzer, Zurich; Dr méd. H. Hengartner, St-Gall; Prof. Dr méd. T. Kuehne, Bâle; Prof. Dr méd. Claudia Kuehni, Registre suisse du cancer de l'enfant, Berne; Prof. Dr méd. K. Leibundgut, Berne; Prof. Dr méd. F. Niggli, Zurich; Dr méd. A. Ansari, Genève; PD Dr méd. J. Rischewski, Lucerne; Prof. Dr méd. N. von der Weid, Bâle.

\section{Registre suisse du cancer de l'enfant}

Dr méd. Elisabeth Kiraly, codage médical; Prof. Dr méd. Claudia Kuehni, directrice; Prof. Dr méd Gisela Michel, directrice remplaçante; MSc Vera Mitter, coordinatrice du projet; Dr. Corina Rueegg, Swiss Childhood Cancer Survivor Study; Marlen Spring, assistante de recherche; Dr Ben Spycher, études étiologiques; Priska Wölfli, informatique.

\section{Références}

1 Michel G, von der Weid NX, Zwahlen M, Adam M, Rebholz CE, et al. (2007) The Swiss Childhood Cancer Registry: rationale, organisation and results for the years 2001-2005. Swiss Med Wkly 137: 502-509.

2 Kuehni C, Von der Weid N (2008) Das Schweizer Kinderkrebsregister als erstes nationales Krebsregister. Schweiz Ärztezeitung 89: 117-119.

3 Wyss N, Pury P, Strippoli M, Lutz J, Bouchardy C, et al. (2011) Krebs in der Schweiz - Stand und Entwicklung von 1983 bis 2007. Neuchâtel, Bundesamt für Statistik (BFS).

4 Heusser R, Noseda G (2012) Rahmenbedingungen für ein wirksames Krebsmonitoring in der Schweiz. Schweizer Krebsbulletin 32: 143-148.

5 Mitter V, Michel G, Wölfli P, Gianinazzi M, Rüegg C, et al. (2012) Swiss Childhood Cancer Registry: Annual Report 2011/2012. Bern: Swiss Childhood Cancer Registry.

6 Steliarova-Foucher E, Stiller C, Lacour B, Kaatsch P (2005) International Classification of Childhood Cancer, third edition. Cancer 103: 1457-1467.

7 Kuehni CE, Rueegg CS, Michel G, Rebholz CE, Strippoli MP, et al. (2011) Cohort profile: The Swiss Childhood Cancer Survivor Study. Int J Epidemiol.

8 Bleyer WA (1990) The impact of childhood cancer on the United States and the world. CA Cancer J Clin 40: 355-367.

9 Niggli F, Kuehni C, Lamontagne-Mueller I (2012) Seltene Krebserkrankungen - das tägliche Brot der pädiatrischen Onkologie. Schweizer Krebsbulletin 32: 209-210.
10 Gatta G, Zigon G, Capocaccia R, Coebergh JW, Desandes E, et al. (2009) Survival of European children and young adults with cancer diagnosed 1995-2002. Eur J Cancer 45: 992-1005.

11 von der Weid N, Mosimann I, Hirt A, Wacker P, Nenadov Beck M, et al. (2003) Intellectual outcome in children and adolescents with acute lymphoblastic leukaemia treated with chemotherapy alone: age- and sex-related differences. Eur J Cancer 39: 359-365.

12 von der Weid N, Swiss Pediatric Oncology G (2001) Late effects in long-term survivors of ALL in childhood: experiences from the SPOG late effects study. Swiss Med Wkly 131: 180-187.

13 von der Weid N, Wagner H (2003) Organisation of follow-up in paediatric oncology. Eur J Cancer 39: 1150-1154; discussion 1155-1156.

14 Essig S, von der Weid NX, Strippoli MP, Rebholz CE, Michel G, et al. (2012) Health-related quality of life in long-term survivors of relapsed childhood acute lymphoblastic leukemia. PLoS One 7: e38015.

15 Michel G, Rebholz CE, von der Weid NX, Bergstraesser E, Kuehni CE (2010) Psychological distress in adult survivors of childhood cancer: the Swiss Childhood Cancer Survivor study. J Clin Oncol 28: 1740-1748.

16 Kuehni CE, Strippoli MP, Rueegg CS, Rebholz CE, Bergstraesser E, et al. (2012) Educational achievement in Swiss childhood cancer survivors compared with the general population. Cancer 118: 1439-1449.

17 Rueegg CS, Michel G, Wengenroth L, von der Weid NX, Bergstraesser E, et al. (2012) Physical performance limitations in adolescent and adult survivors of childhood cancer and their siblings. PLoS One 7: e47944.

18 Michel G, Kuehni CE, Rebholz CE, Zimmermann K, Eiser C, et al. (2011) Can health beliefs help in explaining attendance to follow-up care? The Swiss childhood cancer survivor study. Psychooncology 20: 1034-1043.

19 Rebholz CE, von der Weid NX, Michel G, Niggli FK, Kuehni CE, et al. (2011) Follow-up care amongst long-term childhood cancer survivors: a report from the Swiss Childhood Cancer Survivor Study. Eur J Cancer 47: 221-229.

20 Spycher BD, Feller M, Zwahlen M, Roosli M, von der Weid NX, et al. (2011) Childhood cancer and nuclear power plants in Switzerland: a census-based cohort study. Int J Epidemiol 40: 1247-1260.

21 Aydin D, Feychting M, Schuz J, Tynes T, Andersen TV, et al. (2011) Mobile phone use and brain tumors in children and adolescents: a multicenter case-control study. J Natl Cancer Inst 103: 1264-1276.

22 Kaatsch P, Spix C (2012) German Childhood Cancer Registry - Annual Report 2011 (1980-2010). Mainz: Institute of Medical Biostatistics, Epidemiology and Informatics (IMBEI) at the University Medical Center of the Johannes Gutenberg University.

23 Lacour B, Guyot-Goubin A, Guissou S, Bellec S, Desandes E, et al. (2010) Incidence of childhood cancer in France: National Children Cancer Registries, 2000-2004. Eur J Cancer Prev 19: 173-181.

24 Kroll ME, Murphy MF, Carpenter LM, Stiller CA (2011) Childhood cancer registration in Britain: capturerecapture estimates of completeness of ascertainment. Br J Cancer 104: 1227-1233. 